\title{
THE FORMALISED DEFINITION OF THE PICRIDO HIERACIOIDIS-CIRSIETUM CANDELABRI ASSOCIATION
}

\author{
Nenad Jasprica ${ }^{1 *}$, Milenko Milović ${ }^{2}$ \& Marija PandžA ${ }^{3}$ \\ ${ }^{1}$ Institute for Marine and Coastal Research, University of Dubrovnik, P.O. Box 83, \\ HR-20000 Dubrovnik, Croatia \\ 2“Antun Vrančić" Grammar School, Put Gimnazije 64, HR-22000 Šibenik, Croatia \\ (e-mail: milenko.milovic@si.t-com.hr) \\ ${ }^{3}$ Primary School Murterski škoji, Put škole 8, HR-22243 Murter, Croatia \\ (e-mail: marija.pandza@si.t-com-hr)
}

Jasprica, N., Milović, M. \& Pandža, M., The formalised definition of the Picrido hieracioidisCirsietum candelabri association. Nat. Croat., Vol. 25, No. 2, 331-332, 2016, Zagreb.

The paper gives a formalised description of the Picrido hieracioidis-Cirsietum candelabri association, a new ruderal syntaxon from Croatia.

Key words: Artemisietea vulgaris, new association, description, Croatia.

Jasprica, N., Milović, M. \& Pandža, M., Formalni opis asocijacije Picrido hieracioidis-Cirsietum candelabri. Nat. Croat., Vol. 25, No. 2, 331-332, 2016, Zagreb.

$\mathrm{U}$ radu je prikazan formaliziran opis asocijacije Picrido hieracioidis-Cirsietum candelabri, novog sintaksona u Hrvatskoj.

Ključne riječi: Artemisietea vulgaris, nova asocijacija, opis, Hrvatska.

\section{INTRODUCTION}

The Picrido hieracioidis-Cirsietum candelabri association was proposed by JAsPRICA et al. (2015) with an original diagnosis (table 1, holotypus relevé no. 4, op. cit.), but in a peerreviewed online-only journal (Art. 1). Here we perform the formal validation of this association according to ICPN (WEBER et al., 2000).

\section{NOMENCLATURE}

Plant taxa nomenclature follows Flora Croatica Database [http://hirc.botanic.hr/fcd; Nikolić (2015), accessed on August 20, 2015]. Syntaxonomical nomenclature follows Biondi et al. (2014).

\section{RESULTS AND CONCLUSION}

\section{Description of the syntaxon}

Picrido hieracioidis-Cirsietum candelabri Jasprica, Milović \& Pandža 2016, ass. nov. hoc loco

\footnotetext{
* corresponding author: nenad.jasprica@unidu.hr
} 
Nomenclature type relevé (holotypus hoc loco designatus): relevé NJ-CRO 11, Croatia, Dalmatia, Split-Dalmatia County, municipality of Cista Provo, village of Aržano, along the D39 state road; geographic coordinates: $X=5660995, Y=4828286$ (UTM XJ); altitude: $717 \mathrm{~m}$ a.s.l.; slope: $45^{\circ}$; aspect: S; plot: $6 \mathrm{~m} \times 5 \mathrm{~m}$; vascular plant cover: $90 \%$; average vegetation height: $2 \mathrm{~m}$; substrate: shallow calcareous soils; number of taxa: 50 ; sampled by Nenad Jasprica, Milenko Milović and Marija Pandža on 08-08-2015; sampling scale: 7-grade scale of BrAUn-Blanquet (1964).

Vascular plants composition: Char. Ass.: Cirsium candelabrum 4, Picris hieracioides 1; Dauco carotae-Melilotion albi: Daucus carota 1, Melilotus albus +, Reseda lutea +; Onopordetalia acanthii, Artemisietea vulgaris: Elymus repens 2, Tussilago farfara +, Diplotaxis tenuifolia +, Haplophyllum patavinum +, Ambrosia artemisiifolia +, Silene latifolia ssp. alba r; Companions: Festuco valesiacae-Brometea erecti: Dorycnium pentaphyllum 1, Scabiosa triandra 1, Sanguisorba minor ssp. muricata +, Centaurea spinosociliata ssp. cristata + , Hypericum perforatum +, Medicago lupulina + , Asperula aristata ssp. scabra + , Campanula sibirica +, Linum tenuifolium +, Carlina vulgaris +, Arenaria leptoclados +, Satureja montana +, Medicago prostrata + , Centaurea weldeniana + , Bromus erectus + , Euphorbia cyparissias + , Desmazeria rigida + , Centaurea rupestris $\mathrm{r}$, Stachys recta $\mathrm{r}$, Plantago holosteum $\mathrm{r}$, Sedum sexangulare r, Lactuca viminea r; Stellarietea mediae: Silene vulgaris ssp. angustifolia + , Lactuca serriola +, Fallopia convolvulus +, Bromus sterilis +, Geranium purpureum +, Atriplex patula +, Cirsium arvense +, Conyza canadensis r; Querco-Fagetea sylvaticae: Ulmus minor juv. +; Thlaspietea rotundifolii: Scrophularia canina 1; Trifolio medii-Geranietea sanguinei: Arabis turrita + , Cruciata glabra +; Molinio-Arrhenatheretea: Dactylis glomerata ssp. glomerata + , Plantago altissima r; Galio aparines-Urticetea dioicae: Cruciata laevipes +; Salicetea purpureae: Salix purpurea +; Other: Koeleria sp. + .

Ecology: The association occurs in area within sub-Mediterranean climate, along roadsides mostly on freshly filled deposits of construction waste. It occupies intensively sunny and extremely dry habitats. The stands have optimal development in August when the habitus of the dominant species make them particularly visible. The association is characterized by a clear dominance of hemicryptophytes, and the taxa of South European and Mediterranean origin.

This is a new ruderal association in Croatia. It belongs to the class Artemisietea vulgaris Lohmeyer, Preising \& Tüxen ex von Rochow 1951, the order Onopordetalia acanthii BraunBlanquet \& Tüxen ex Klika in Klika \& Hadač 1944 and the alliance Dauco carotae-Melilotion albi Görs ex Rostański \& Gutte 1971.

Received October 26, 2016

\section{REFERENCES}

Biondi, E., Blasi, C., Allegrezza, M., Anzellotti, I., Azzella, M. M., Carli, E. et al., 2014: Plant communities of Italy: The Vegetation Prodrome. Plant Biosystem 148, 728-814.

Braun-Blanquet, J., 1964: Pflanzensoziologie. Grundzüge der Vegetationskunde [Plant sociology. Basic course of Vegetation Science]. 3. New York, USA: Springer-Verlag.

Jasprica, N., Milović, M. \& PandžA, M., 2015: Picrido hieracioidis-Cirsietum candelabri Jasprica et al. 2015 - a ruderal association new to Croatia. Glasnik Hrvatskog botaničkog društva 3(2), 4-14.

Nikolić, T. (ed.), 2015: Flora Croatica baza podataka [Flora Croatica database] (online). Available: http:// hirc.botanic.hr/fcd. Botanički zavod, Prirodoslovno-matematički fakultet, Sveučilište u Zagrebu. [last accessed August 20, 2015].

Weber, H.E., Moravec, J. \& Theurillat, J.P., 2000: International code of phytosociological nomenclature. 3rd edition. Journal of Vegetation Science 11, 739-768. 